\title{
Approximate results for rainbow labelings
}

\author{
Anna Lladó · Mirka Miller
}

Received: date / Accepted: date

Abstract A simple graph $G=(V, E)$ is said to be antimagic if there exists a bijection $f: E \rightarrow[1,|E|]$ such that the sum of the values of $f$ on edges incident to a vertex takes different values on distinct vertices. The graph $G$ is distance antimagic if there exists a bijection $f: V \rightarrow[1,|V|]$, such that $\forall x, y \in V$,

$$
\sum_{x_{i} \in N(x)} f\left(x_{i}\right) \neq \sum_{x_{j} \in N(y)} f\left(x_{j}\right)
$$

Using the polynomial method of Alon we prove that there are antimagic injections of any graph $G$ with $n$ vertices and $m$ edges in the interval [1,2n+m-4] and, for trees with $k$ inner vertices, in the interval $[1, m+k]$. In particular, a tree all of whose inner vertices are adjacent to a leaf is antimagic. This gives a partial positive answer to a conjecture by Hartsfield and Ringel.

We also show that there are distance antimagic injections of a graph $G$ with order $n$ and maximum degree $\Delta$ in the interval $[1, n+t(n-t)]$, where $t=$ $\min \{\Delta,\lfloor n / 2\rfloor\}$, and, for trees with $k$ leaves, in the interval $[1,3 n-4 k]$. In particular, all trees with $n=2 k$ vertices and no pairs of leaves share their neighbour are distance antimagic, a partial solution to a conjecture of Arumugam.

Keywords Graph labeling · Polynomial method

Mirka Miller passed away on January 5th 2016 while this paper was under review.

Anna Lladó

Department of Mathematics

Univ. Politècnica de Catalunya, Barcelona, Spain

E-mail: aina.llado@upc.edu

Mirka Miller

School of Mathematical and Physical Sciences, University of Newcastle, Australia; and Department of Mathematics, University of West Bohemia, Pilsen, Czech Republic

E-mail: Mirka.Miller@newcastle.edu.au 


\section{Introduction}

In this paper we shall consider two kinds of labelings: antimagic and distance antimagic. The concept of an antimagic labeling of a graph was introduced by Hartsfield and Ringel in 1990 [13].

In general, an edge labeling of a graph $G=(V, E)$ is a bijection $l: E \rightarrow$ $\{1,2, \ldots,|E|\}:=[1,|E|]$. The weight of a vertex $v, w t(v)$, is the sum of the labels of all edges incident to $v$.

An edge labeling $l$ of $G$ is called antimagic if all vertex weights in $G$ are pairwise distinct. A graph $G$ is said to be antimagic if it has an antimagic labeling.

Hartsfield and Ringel [13] showed that each path $P_{m}$, star $S_{m}$, cycle $C_{m}$, complete graph $K_{m}$, wheel $W_{m}$ and bipartite graph $K_{2, m}, m \geq 3$, is antimagic. They further conjectured

Conjecture 1 (Harstfield-Ringel, 1990) Every connected graph $G \neq K_{2}$ is antimagic.

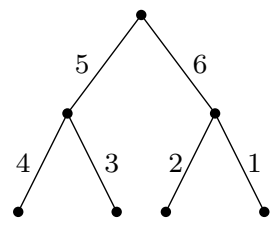

Fig. 1 An antimagic labeling of a tree.

Over the period of more than two decades, many attempts have been made to settle the conjecture. While in general the Hartsfield and Ringel conjecture remains open, some partial results are known which support the conjecture. Alon et al. [3] used probabilistic methods and some techniques from analytic number theory to show that the conjecture is true for all graphs having minimum degree at least $\Omega(\log |V(G)|)$. They also proved that if $G$ is a graph with order $|V(G)| \geq 4$ and maximum degree $\Delta(G),|V(G)|-2 \leq \Delta(G) \leq|V(G)|-1$, then $G$ is antimagic, and that all complete multipartite graphs, except $K_{2}$, are antimagic. Cranston [11] proved that every regular bipartite graph (complete or not) is antimagic. Hefetz [14] used the Combinatorial Nullstellensatz to prove that a graph with $3^{k}$ vertices, where $k$ is a positive integer, which admits a $K_{3}$-factor is antimagic. Various papers on the antimagicness of particular classes of graphs have been published, for example, see [9,19-21]. For more details on antimagic labeling for particular classes of graphs see the dynamic survey [12], see also [5].

There is now a great wealth of evidence in support of the conjecture. However a full general proof still eludes us. Even the weaker conjecture, that every tree different from $K_{2}$, is antimagic still remains open. The most general result for trees is due to Kaplan, Lev and Roditty [15] who proved that every tree with at most one vertex of degree 2 is antimagic. See also $[8,13]$ for other results on antimagic trees.

A less known but closely related type of graph labeling known as the "distance antimagic labeling", or more precisely, the "1-distance vertex antimagic vertex labeling", has been defined as follows. 


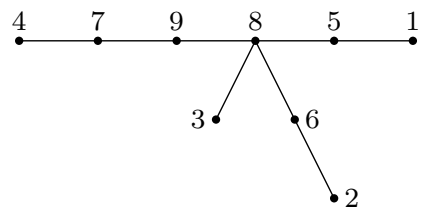

Fig. 2 A distance antimagic labeling of a tree.

\section{Definition 1 (Miller, Rodger, Simantujak, 2003)}

A distance antimagic labeling of a graph $G=(V, E)$ is a bijection $f: V \rightarrow$ $[1,|V|]$, such that for every pair $x, y$ of distinct vertices,

$$
\sum_{u \in N(x)} f(u) \neq \sum_{u \in N(y)} f(u) .
$$

An obvious necessary condition for $G$ to be distance antimagic is

$$
N(x) \neq N(y), \text { for each } x, y \in V(G) .
$$

Paths $P_{n}$, cycles $C_{n}$, wheels $W_{n \neq 4}$, regular bipartite graphs and some particular caterpillars are examples of graphs which have been proved to be distance antimagic. Arumugam [4] has conjectured that all trees satisfying the obvious necessary condition are distance antimagic.

Conjecture 2 (Arumugam, 2012) A tree $T$ is distance antimagic if and only if every vertex is adjacent to at most one leaf.

In this paper we give approximate results to the two above conjectures, that is, we find upper bounds for the smallest integer such that there is an injection with the corresponding rainbow property. An analogous approach has been also considered for other kinds of labelings; see e.g., Bollobás and Pikhurko [6] or Lladó, López and Moragas [17].

We start with distance antimagic graph labeling. For a given class $\mathcal{X}$ of graphs, let $D A(\mathcal{X}, n)$ denote the smallest integer $N$ such that, for each graph $G \in \mathcal{X}$ of order $n$, there is an injection $f: V \rightarrow[1, N]$ such that the sums

$$
\sum_{y \in N(x)} f(y), x \in V
$$

are pairwise distinct. We call such a map a distance antimagic injection.

Let $\mathcal{G}$ be the class of all graphs which have no two vertices with the same neighborhood and let $G \in \mathcal{G}$ with order $n$. For any ordering $v_{1}, \ldots, v_{n}$ of the vertices of $G$ the map $f\left(v_{i}\right)=2^{i}$ is clearly a distance antimagic injection in $G$. Thus we have $D A(\mathcal{G}, n) \leq 2^{n}$. This trivial exponential bound can be reduced for general graphs.

Theorem 1 Let $\mathcal{G}_{\Delta}$ be the class of graphs in $\mathcal{G}$ with maximum degree $\Delta$. Then

$$
D A\left(\mathcal{G}_{\Delta}, n\right) \leq n+t(n-t), \text { where } t=\min \{\Delta,\lfloor n / 2\rfloor\} .
$$


A better bound can be obtained for trees.

Theorem 2 Let $\mathcal{T}_{k}$ be the class of trees in $\mathcal{G}$ which have $k$ leaves. Then

$$
D A\left(\mathcal{T}_{k}, n\right) \leq 3 n-4 k \text {. }
$$

In particular, all trees in $\mathcal{T}_{k}$ with $n=2 k$ vertices are distance antimagic.

In particular, for an arbitrary tree $T$, the tree obtained from $T$ by attaching one leaf to each vertex of $T$ is distance antimagic.

Similar definitions can be made for antimagic labelings. Let $A(\mathcal{X}, m)$ denote the smallest integer $N$ such that, for each graph $G \in \mathcal{X}$ with $m$ edges, there is an injection $f: E(G) \rightarrow[1, N]$ such that the sums

$$
\sum_{y x \in E} f(y), x \in V
$$

are pairwise distinct. We call such a map $f$ an antimagic injection. As in the distance antimagic case, if $e_{1}, \ldots, e_{m}$ are the edges of $G$ and $m>1$ then the map $f\left(e_{i}\right)=2^{i}$ is clearly an antimagic injection, so $A(\mathcal{X}, m) \leq 2^{m}$. We show

Theorem 3 Every graph $G$ with $m>1$ edges and $n$ vertices admits an antimagic injection on $[1,2 n+m-4]$.

For trees the upper bound can be reduced. Recall that the base tree of a tree $T$ is obtained from $T$ by removing all its leaves. A vertex of $T$ is said to be an inner vertex of a subtree $T^{\prime}$ of $T$ if all its neighbours in $T$ belong to $T^{\prime}$.

Theorem 4 Let $\mathcal{T}_{k}^{\prime}$ denote the class of trees whose base tree has $k$ inner vertices. For $m>1$ we have $A\left(\mathcal{T}_{k}^{\prime}, m\right) \leq m+k$.

In particular, a tree different from $K_{2}$ whose base tree has no inner vertices is antimagic.

It follows from the last statement in Theorem 4 that, for an arbitrary tree $T$, the tree obtained from $T$ by attaching one leaf to each vertex of $T$ is antimagic.

The proofs of the above theorems use the polynomial method of Alon (Combinatorial Nullstellensatz) which we recall next.

Theorem 5 (Combinatorial Nullstellensatz, Alon (1999) [1])

Let $P\left(x_{1}, \ldots, x_{k}\right)$ be a polynomial of degree $d$ in $F\left[x_{1}, \ldots, x_{k}\right]$ over a field $F$. Let $S_{1}, \ldots, S_{k}$ be subsets of $F$ with $\left|S_{i}\right|>d_{i} \geq 0$ such that $\sum_{i=1}^{k} d_{i}=d$.

If the coefficient of the monomial $\prod_{i=1}^{k} x_{i}{ }^{d_{i}}$ in $f$ is nonzero, then there exists

$$
\left(s_{1}, \ldots, s_{k}\right) \in S_{1} \times \cdots \times S_{k}
$$

such that $P\left(s_{1}, \ldots, s_{k}\right) \neq 0$.

The proofs of Theorems 1 and 2 are given in Section 2, whereas Section 3 contains the proofs of Theorems 3 and 4 . The last section contains some final remarks. 


\section{Distance antimagic injections}

As mentioned before, the proofs use the polynomial method. For a set $x_{1}, \ldots, x_{n}$, we denote by $V\left(x_{1}, \ldots, x_{n}\right)$ the Vandermonde polynomial

$$
V\left(x_{1}, \ldots, x_{n}\right)=\prod_{1 \leq i<j \leq n}\left(x_{i}-x_{j}\right)
$$

We recall that the Vandermonde polynomial has an expansion of the form

$$
V\left(x_{1}, \ldots, x_{n}\right)=\sum_{\sigma \in \operatorname{Sym}(n)}(-1)^{\operatorname{sgn}(\sigma)} x_{\sigma(1)}^{n-1} x_{\sigma(2)}^{n-2} \cdots x_{\sigma(n)}^{0},
$$

and $V\left(a_{1}, \ldots, a_{n}\right) \neq 0$ if and only if the $a_{i}$ 's are pairwise distinct.

The proof of Theorem 1 is a quite straightforward application of the polynomial method and it is included to illustrate the technique.

Proof of Theorem 1. Let $G \in \mathcal{G}_{\Delta}$ with order $n$. Let $v_{1}, \ldots, v_{n}$ be the vertices of $G$. Let $x_{1}, \ldots, x_{n}$ be variables and, for each $i$, define

$$
y_{i}=\sum_{v_{j} \in N\left(v_{i}\right)} x_{j} .
$$

Consider the polynomial $P \in \mathbb{R}\left[x_{1}, \ldots, x_{n}\right]$ defined as

$$
P\left(x_{1}, \ldots, x_{n}\right)=V\left(x_{1}, \ldots, x_{n}\right) V\left(y_{1}, \ldots, y_{n}\right) .
$$

A map $f: V \rightarrow \mathbb{N}$ is a distance antimagic injection if and only if

$$
P\left(f\left(v_{1}\right), \ldots, f\left(v_{n}\right)\right) \neq 0 .
$$

Since $G$ contains no two vertices with the same neighborhood, it admits distance antimagic injections, so that $P$ is not the zero polynomial.

On the other hand, considering a term $\left(y_{j}-y_{k}\right), x_{i}$ will appear in the term if it is present in exactly one of $y_{j}, y_{k}$. Hence the variable $x_{i}$ appears at most $t(n-t)$ times in $V\left(y_{1}, \ldots, y_{n}\right)$, where $t=\min \{\Delta,\lfloor n / 2\rfloor\}$. Therefore, every monomial $x_{1}^{\alpha_{1}} \cdots x_{n}^{\alpha_{n}}$ in $P$ with nonzero coefficient satisfies

$$
\max \alpha_{i} \leq(n-1)+t(n-t) .
$$

It follows from the Combinatorial Nullstellensatz that, by choosing

$$
S_{1}=\ldots=S_{n}=[1, n+t(n-t)],
$$

there are $1 \leq a_{1}, \ldots, a_{n} \leq n+t(n-t)$ such that $P\left(a_{1}, \ldots, a_{n}\right) \neq 0$. Thus the assignment $f\left(v_{i}\right)=a_{i}$ gives a distance antimagic injection.

The proof of Theorem 2 involves a more efficient use of the polynomial method. We recall that $\left(V\left(x_{1}, \ldots, x_{n}\right)\right)^{2}$ has a term of the form

$$
x_{1}^{n-1} \cdots x_{n}^{n-1},
$$

with coefficient $n$ ! up to a sign (see e.g. Alon [2].) An analogous result holds for the fourth power of the Vandermonde polynomial. This is a consequence of the Dyson conjecture of which we next reproduce the statement from Karasev and Petrov [16], where a simple proof with the Combinatorial Nullstellensatz can be found. 


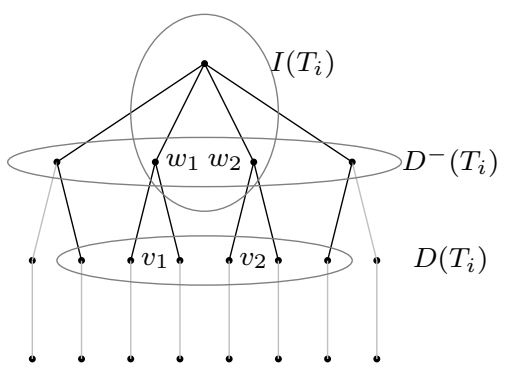

Fig. 3 Illustration of the notation in the proof of Theorem 2.

Theorem 6 ([16]) Let $\alpha_{1}, \ldots, \alpha_{n}$ be positive integers and $\alpha=\sum_{i} \alpha_{i}$. Denote by $c$ be the coefficient of

$$
x_{1}^{\alpha-\alpha_{1}} \cdots x_{n}^{\alpha-\alpha_{n}}
$$

in the polynomial

$$
\prod_{1 \leq i<j \leq n}(-1)^{a_{j}}\left(x_{j}-x_{i}\right)^{a_{i}+a_{j}}
$$

Then

$$
c=\frac{\alpha}{\alpha_{1} ! \cdots \alpha_{n} !} .
$$

By choosing $\alpha_{1}=\cdots=\alpha_{n}=2$ in Theorem 6 we get

Corollary 1 The coefficient of $x_{1}^{2(n-1)} \cdots x_{n}^{2(n-1)}$ in $\left(V\left(x_{1}, \ldots, x_{n}\right)\right)^{4}$ is,

$$
\frac{(2 n) !}{2^{n}}
$$

Using Corollary 1 we next prove Theorem 2.

Proof of Theorem 2. Let $T$ be a tree in $\mathcal{T}_{k}$. For a subtree $T^{\prime} \subseteq T$ we denote by

$$
I\left(T^{\prime}\right)=\left\{v \in V\left(T^{\prime}\right): N(v) \subseteq V\left(T^{\prime}\right)\right\}
$$

the set of inner vertices in $T^{\prime}$ (its full neighborhood in $T$ is contained in $V\left(T^{\prime}\right)$ ) and by

$$
D\left(T^{\prime}\right)=\left\{v \in V\left(T^{\prime}\right):\left|N_{T^{\prime}}(v)\right|=1\right\},
$$

the set of leaves of $T^{\prime}$. We also let

$$
D^{-}\left(T^{\prime}\right)=\left\{N_{T^{\prime}}(v): v \in D\left(T^{\prime}\right)\right\}
$$

denote the set of vertices in $T^{\prime}$ which are adjacent to some leaf of $T^{\prime}$. Let $T_{0}=T$ and for $i \geq 1$ let $T_{i}=T_{i-1}-D\left(T_{i-1}\right)$ be the subtree of $T$ obtained from $T_{i-1}$ by deleting its leaves. In this way we obtain a monotone decreasing chain

$$
T=T_{0} \supset T_{1} \supset T_{2} \supset \cdots \supset T_{l},
$$

where $V\left(T_{i}\right)=V\left(T_{i+1}\right) \cup D\left(T_{i}\right)$ and $T_{l}$ is the center of $T$ consisting of a single vertex or a single edge. Figure 3 illustrates the notation. 
We will define a distance antimagic injection of $T$ level by level starting from $T_{l}$. We label the vertices in $T_{l}$ with $\{1\}$ (if $T_{l}$ consists of one vertex) or with $\{1,2\}$ (if it consists of two vertices.)

Suppose that $f$ has been defined on $V\left(T_{i+1}\right)$ satisfying the following three properties:

(i) $f$ is an injection on $V\left(T_{i+1}\right)$;

(ii) With the convention $S(v)=\sum_{u \in N(v)} f(u)$, the neighbour sums in

$$
S\left(I\left(T_{i+1}\right)\right)=\left\{S(v): v \in I\left(T_{i+1}\right)\right\}
$$

of the inner vertices in $T_{i+1}$ are pairwise distinct;

(iii) if $v \in D^{-}(T) \cap V\left(T_{i+1}\right)$ then $f(v) \notin S\left(I\left(T_{i+1}\right)\right)$.

These three properties are trivially satisfied if $i+1=l$. We will extend $f$ to $T_{i}$ in two steps by preserving the three above properties.

Let $w_{1}, \ldots, w_{r}$ be the vertices in $I\left(T_{i}\right) \backslash I\left(T_{i+1}\right)$, the new inner vertices in $T_{i}$, which do have neighbors in $D\left(T_{i}\right)$. Then, since $T$ has no pair of leaves adjacent to the same vertex,

$$
r \leq\left|D\left(T_{i}\right)\right| \leq|D(T)|=k .
$$

For each $w_{j}$ choose one neighbor $v_{j} \in N\left(w_{j}\right) \cap D\left(T_{i}\right)$. Label the vertices in $D\left(T_{i}\right) \backslash$ $\left\{v_{1}, \ldots, v_{r}\right\}$ with pairwise distinct numbers in the set

$$
\left[1, N_{i}^{\prime}\right] \backslash\left(f\left(V\left(T_{i+1}\right)\right) \cup S\left(I\left(T_{i+1}\right)\right)\right),
$$

where $N_{i}^{\prime}=\left|V\left(T_{i}\right)\right|+\left|I\left(T_{i+1}\right)\right|$ (this is possible since this set contains at least $\left|D\left(T_{i}\right)\right|$ elements.) Therefore, $f$ is still injective and no vertex in $D^{-}(T)$ has received a value in $S\left(I\left(T_{i+1}\right)\right)$.

If $r=0$ then $f$ has been extended to $T_{i}$ as desired. Suppose that $r>0$. Let

$$
S^{\prime}\left(w_{j}\right)=\sum_{u \in N\left(w_{j}\right) \backslash v_{j}} f(u), j=1, \ldots, r,
$$

and consider the following polynomials in $\mathbb{R}\left[x_{1}, \ldots, x_{k}\right]$ :

$$
\begin{aligned}
Q_{1, i} & =V\left(x_{1}, \ldots, x_{r}\right) \prod_{j=1}^{r} \prod_{u \in V\left(T_{i}\right) \backslash\left\{v_{1}, \ldots, v_{r}\right\}}\left(x_{j}-f(u)\right) ; \\
Q_{2, i} & =V\left(x_{1}+S^{\prime}\left(w_{1}\right), \ldots, x_{r}+S^{\prime}\left(w_{r}\right)\right) \prod_{j=1}^{r} \prod_{u \in I\left(T_{i+1}\right)}\left(x_{j}+S^{\prime}\left(w_{j}\right)-S(u)\right) ; \\
Q_{3, i} & =\prod_{1 \leq i<j \leq r}\left(x_{i}-\left(S^{\prime}\left(w_{j}\right)+x_{j}\right)\right) \prod_{j=1}^{k} \prod_{u \in I\left(T_{i+1}\right)}\left(x_{j}-S(u)\right) .
\end{aligned}
$$

We observe that

$$
\begin{aligned}
V\left(x_{1}+S^{\prime}\left(w_{1}\right), \ldots, x_{r}+S^{\prime}\left(w_{r}\right)\right) & =V\left(x_{1}, \ldots, x_{r}\right)+\text { terms of lower degree; and } \\
\prod_{1 \leq i<j \leq r}\left(x_{i}-\left(S^{\prime}\left(w_{j}\right)+x_{j}\right)\right) & =V\left(x_{1}, \ldots, x_{r}\right)+\text { terms of lower degree. }
\end{aligned}
$$


Hence, the polynomial $P_{i}=Q_{1, i} Q_{2, i} Q_{3, i}$ can be written as

$$
P_{i}=\left(V\left(x_{1}, \ldots, x_{r}\right)\right)^{3} \prod_{j=1}^{r} x_{j}^{m_{i}}+\text { terms of lower degree }
$$

where

$$
m_{i}=\left(\left|V\left(T_{i}\right)\right|-r\right)+2\left|I\left(T_{i+1}\right)\right| .
$$

It follows from Corollary 1 that $\left(V\left(x_{1}, \ldots, x_{r}\right)\right)^{3}$ has a monomial with nonzero coefficient whose largest exponent is at most $2(r-1)$. Hence our polynomial $P_{i}$ has a term with largest exponent at most

$$
N_{i}=\left|V\left(T_{i}\right)\right|+2\left|I\left(T_{i+1}\right)\right|+r-2,
$$

whose coefficient is nonzero.

By Theorem 5 there are $1 \leq a_{1}, \ldots, a_{r} \leq N_{i}+1$ such that $P_{i}$ takes a nonzero value on $\left(a_{1}, \ldots, a_{k}\right)$. Define $f\left(u_{i}\right)=a_{i}, i=1, \ldots, r$. Let us check that in this way we have extended $f$ to $V\left(T_{i}\right)$ by preserving properties (i)-(iii).

The way $f$ has been defined on $V\left(T_{i}\right) \backslash\left\{v_{1}, \ldots, v_{r}\right\}$ and the fact that $Q_{1, i}$ is nonzero on $\left(a_{1}, \ldots, a_{r}\right)$ ensure that $f$ is injective on $V\left(T_{i}\right)$, yielding property (i). Since no vertex in $D\left(T_{i}\right) \backslash\left\{v_{1}, \ldots, v_{r}\right\}$ is an inner vertex of $T_{i}$, the fact that $Q_{2, i}\left(a_{1}, \ldots, a_{k}\right) \neq 0$ ensures that the values $S\left(w_{j}\right)=S^{\prime}\left(w_{j}\right)+a_{j}, 1 \leq j \leq r$, are pairwise distinct and different from the values of $S(u)$ for $u \in I\left(T_{i+1}\right)$. Moreover, if $i=0$, then the values $S\left(v_{j}\right)=f\left(w_{j}\right)$ are pairwise distinct (by property (i) of $f$ on $V\left(T_{1}\right)$ ) and different from the values $\left\{S(u): u \in I\left(T_{1}\right)\right\}$ (by property (iii) of $f$ on $T_{1}$ ), which gives property (ii). Finally, the fact that $Q_{3, i}\left(a_{1}, \ldots, a_{r}\right) \neq 0$ ensures that $\left\{f\left(v_{j}\right), 1 \leq j \leq r\right\}$ is disjoint with

$$
\left\{S(u): u \in I\left(T_{i+1}\right)\right\} \cup\left\{S\left(w_{1}\right), \ldots, S\left(w_{k}\right)\right\}=\left\{S(u): u \in I\left(T_{i}\right) \backslash D\right\} .
$$

Together with the way $f$ has been defined on $V\left(T_{i}\right) \backslash\left\{v_{1}, \ldots, v_{r}\right\}$, this ensures that the label of every vertex in $D^{-}(T)$ does not coincide with any neighbor sum, providing property (iii).

We note that $N_{i}$ is larger than the $N_{i}^{\prime}$ (1) defined in the first part of the $i$-th step, unless $r=1$ and $\left|I\left(T_{i+1}\right)\right|=0$, in which case we define $N_{i}=N_{i}^{\prime}=\left|V\left(T_{i}\right)\right|$. In either case, $f$ can be defined on $T_{i}$ by using elements in $\left[1, N_{i}\right]$.

For $i \geq 1$, the above procedure produces an injection $f$ on $\left[1, N_{i}+1\right]$, where $N_{i}$ is defined in (2). We observe that in the last step, when $i=0$, by using the notation as in the above procedure, we have $r=k,\left\{w_{1}, \ldots, w_{k}\right\}=D^{-}(T)$ and $\left\{v_{1}, \ldots, v_{k}\right\}=D(T)$. In this case the polynomial $Q_{3,0}$ which ensures that the labels given to vertices of $D^{-}(T)$ do not coincide with neighbor sums, is no longer required since none of the new vertices $v_{1}, \ldots, v_{k}$ belongs to $D^{-}(T)$. Hence the last step can be simplified to just consider the polynomial

$$
Q_{0}=Q_{1,0} Q_{2,0}=\left(V\left(x_{1}, \ldots, x_{k}\right)\right)^{2} \prod_{j=1}^{k} x_{j}^{m_{0}},
$$

where $m_{0}=|V(T)|-k+\left|I\left(T_{1}\right)\right|=n-k+(n-2 k)=2 n-3 k$ (we here recall that, since $T \in \mathcal{T}_{k}$, we have $\left|I\left(T_{1}\right)\right|=\left|I\left(T_{2}\right)\right|=n-2 k$.) Moreover, the polynomial $Q_{0}$ has a term with nonzero coefficient and all the exponents equal to $N_{0}=(k-1)+m_{0}=$ 
$2(n-k)-1$. Therefore the last extension of $f$ to $V\left(T_{0}\right)=V(T)$ can be performed in the interval $[1,2(n-k)]$.

When the process finishes at $i=0$, the properties (i)-(iii) ensure that $f$ is a distance antimagic injection taking values in the interval $\left[1, \max _{i} N_{i}+1\right]$. Since $\max _{i} N_{i}=\max \left\{N_{1}, N_{0}\right\}$ and

$$
N_{1} \leq\left|V\left(T_{1}\right)\right|+2\left|I\left(T_{2}\right)\right|+k-2 \leq(n-k)+2(n-2 k)+k-2=3 n-4 k-2,
$$

we have $\max \left\{N_{1}, N_{0}\right\} \leq 3 n-4 k-1$. It follows that $f$ can be defined in all cases in the interval $[1,3 n-4 k]$. This completes the proof.

\section{Antimagic injections}

The proofs of Theorems 3 and 4 are analogous to the proofs of Theorems 1 and 2 respectively, but they are somewhat simpler.

Proof of Theorem 3. Let $G$ be a graph with order $n$ and $m$ edges. Let $v_{1}, \ldots, v_{n}$ be the vertices of $G$ and let $e_{1}, \ldots, e_{m}$ be the edges of $G$. For each vertex $v_{i}$ denote by $e\left(v_{i}\right)$ the set of edges incident with $v_{i}$. Let $x_{1}, \ldots, x_{m}$ be variables and, for each $i=1, \ldots, m$, define

$$
y_{i}=\sum_{e_{j} \in e\left(v_{i}\right)} x_{j} .
$$

Consider the polynomial $P \in \mathbb{R}\left[x_{1}, \ldots, x_{m}\right]$ defined as

$$
P\left(x_{1}, \ldots, x_{m}\right)=V\left(x_{1}, \ldots, x_{m}\right) V\left(y_{1}, \ldots, y_{n}\right) .
$$

A map $f: E \rightarrow \mathbb{N}$ is an antimagic injection if and only if

$$
P\left(f\left(e_{1}\right), \ldots, f\left(e_{m}\right)\right) \neq 0 .
$$

Since $G$ admits antimagic injections, $P$ is not the zero polynomial. We observe that every variable $x_{i}$ appears in at most two different variables $y_{j}$ (the two leaves of the corresponding edge). By looking at the expansion of the Vandermonde polynomials, we see that every monomial

$$
x_{1}^{\alpha_{1}} \cdots x_{m}^{\alpha_{m}}
$$

in $P$ with nonzero coefficient satisfies $\max \alpha_{i} \leq(m-1)+2(n-2)$. It follows from the Combinatorial Nullstellenstaz that there are $1 \leq a_{1}, \ldots, a_{n} \leq 2 n+m-4$ such that $P\left(a_{1}, \ldots, a_{m}\right) \neq 0$. Thus the assignment $f\left(e_{i}\right)=a_{i}$ gives an antimagic injection.

We finally prove Theorem 4.

Proof of Theorem 4. As mentioned before the proof follows the same lines as the proof of Theorem 2. We use the same notation concerning the set $I\left(T^{\prime}\right)$ of inner vertices, the set $D\left(T^{\prime}\right)$ of leaves and $D^{-}\left(T^{\prime}\right)$ the set of vertices adjacent to leaves of a subtree $T^{\prime} \subseteq T$. We again define

$$
T_{0}=T \supset T_{1} \supset \cdots \supset T_{l} .
$$

where $T_{i+1}=T_{i} \backslash D\left(T_{i}\right)$ and now $T_{l}$ is an edge. Suppose that $f$ has been defined on $E\left(T_{i+1}\right)$ injectively and such that the edgesums $S(v)=\sum_{u \in N(v)} f(u v)$ of the 
inner vertices of $T_{i+1}$ are pairwise distinct. This is certainly possible when $i+1=l$ since $T \neq K_{2}$, so at most one vertex in $T_{l}$ is inner. We next extend $f$ to $T_{i}$ such that it is still injective and the edgesums of inner vertices of $T_{i}$ are pairwise distinct.

Let $w_{1}, \ldots, w_{r}$ be the vertices in $I\left(T_{i}\right) \backslash I\left(T_{i+1}\right)$ and, for each $w_{j}$ choose one neighbor $v_{j} \in N\left(w_{j}\right) \cap D\left(T_{i}\right)$. Label the edges in $\left\{u w_{j}: j=1, \ldots, r, u \in N\left(w_{j}\right) \cap D\left(T_{i}\right)\right\} \backslash$ $\left\{v_{j}\right\}$ with pairwise distinct numbers in

$$
\left[1, N_{i}^{\prime}\right] \backslash\left(f\left(E\left(T_{i+1}\right)\right) \cup S\left(I\left(T_{i+1}\right)\right)\right)
$$

where $N_{i}^{\prime}=\left(\left|E\left(T_{i}\right)\right|-r\right)+\left|I\left(T_{i+1}\right)\right|$ (this is possible since this set contains at least $\left|D\left(T_{i}\right)\right|-r$ elements.) In this way, $f$ is still injective. If $r=0$ this provides the desired extension of $f$ to $T_{i}$. Suppose $r>0$.

Let

$$
S^{\prime}\left(w_{j}\right)=\sum_{u \in N\left(w_{j}\right) \backslash v_{j}} f\left(u w_{j}\right), j=1, \ldots, r
$$

and consider the following polynomials in $\mathbb{R}\left[x_{1}, \ldots, x_{r}\right]$ :

$$
\begin{aligned}
& Q_{1, i}=V\left(x_{1}, \ldots, x_{r}\right) \prod_{j=1}^{r} \prod_{e \in E\left(T_{i}\right) \backslash\left\{v_{1} w_{1}, \ldots v_{r} w_{r}\right\}}\left(x_{j}-f(e)\right) ; \\
& Q_{2, i}=V\left(x_{1}+S^{\prime}\left(w_{1}\right), \ldots, x_{r}+S^{\prime}\left(w_{r}\right)\right) \prod_{j=1}^{r} \prod_{u \in I\left(T_{i+1}\right)}\left(x_{j}+S^{\prime}\left(w_{j}\right)-S(u)\right) .
\end{aligned}
$$

We can write

$$
P_{i}=Q_{1, i} Q_{2, i}=\left(V\left(x_{1}, \ldots, x_{r}\right)\right)^{2} \prod_{j=1}^{r} x^{m_{i}}+\text { terms of lower degree, }
$$

where $m_{i}=\left|E\left(T_{i}\right)\right|-r+\left|I\left(T_{i+1}\right)\right|$. Hence $P_{i}$ has a term

$$
x_{1}^{m_{i}+r-1} \cdots x_{r}^{m_{i}+r-1}
$$

with nonzero coefficient. We observe that $N_{i}=\left|E\left(T_{i}\right)\right|+\left|I\left(T_{i+1}\right)\right|-1 \geq N_{i}^{\prime}$. By the Combinatorial Nullstellensatz, there are $1 \leq a_{1}, \ldots, a_{r} \leq N_{i}+1$ such that $P_{i}$ does not vanish in $\left(a_{1}, \ldots, a_{r}\right)$. By defining $f\left(v_{j} w_{j}\right)=a_{j}$ for $j=1, \ldots, r$ we have extended $f$ to $E\left(T_{i}\right)$ injectively and such that the edgesums of inner vertices of $T_{i}$ are pairwise distinct. When $i=0$ we have completed our definition of $f$ taking values on $\left[1, N_{0}+1\right]$, where

$$
N_{0}=|E(T)|+\left|I\left(T_{1}\right)\right|-1=m+k-1 .
$$

This completes the proof. 


\section{Final Remarks}

Alon's polynomial method is a useful tool for proving the existence of labelings of graphs with some prescribed properties. It has however some limitations and its straight application cannot provide, for instance, a proof of the antimagic conjecture, even for trees.

As mentioned in the proof of Theorem 3, a labeling $f: E(T) \rightarrow \mathbb{N}$ of a graph $G$ with vertex set $\left\{v_{1}, \ldots, v_{n}\right\}$ and $m$ edges is antimagic if and only if the polynomial $P\left(x_{1}, \ldots, x_{m}\right)=V\left(x_{1}, \ldots, x_{m}\right) V\left(y_{1}, \ldots, y_{n}\right)$ is nonzero in $\left(f\left(v_{1}\right), \ldots, f\left(v_{n}\right)\right)$, where the variables $y_{i}$ are defined as in the proof. For the simple path $P_{3}$ with three vertices, this polynomial reduces to $P\left(x_{1}, x_{2}\right)=-x_{1}^{3} x_{2}+2 x_{1}^{2} x_{2}^{2}-x_{1} x_{2}^{3}$. Direct application of the Combinatorial Nullstellensatz to this polynomial only ensures that there is an antimagic injection in $\{1,2,3\}$, although this path admits an obvious antimagic labeling. The reason is that the method provides the stronger result that every set with three elements can be used as values of a labeling, and for this stronger statement the minimum cardinality for $P_{3}$ is indeed 3 . However one can exploit the generality of the results obtained with the method in other directions. One example is the modular version of Theorem 4. Let us define a modular antimagic injection $f: E(G) \rightarrow \mathbb{Z}_{n}$ of a graph as an injection such that the edge sums

$$
\left\{\sum_{u \in N(v)} f(u v): v \in V(G)\right\}
$$

are pairwise distinct modulo $n$. In this case we say that $T$ is $n$-antimagic. It is proved in [15] that every tree with $m$ edges and at most one vertex of degree 2 is $(m+1)$-antimagic whenever $m$ is even. The proof of Theorem 4 provides the following modular version.

Theorem 7 Let $T$ be a tree with $p$ edges, $p$ a prime, whose base tree has no inner vertices. Then $T$ is $p$-antimagic.

Proof Replacing the field $\mathbb{R}$ in the proof of Theorem 4 by the finite field $\mathbb{F}_{p}, p$ a prime, the coefficient of the monomials which appear in the applications of the Combinatorial Nullsetellensatz is $r$ ! with $r<p$, which is clearly nonzero in $\mathbb{F}_{p}$.

We finish by noting that there are simple direct arguments which provide approximate results. For instance, it can be proved by simple induction and the pigeonhole principle that every tree with $m \geq 2$ edges admits an antimagic injection in $[1,3 m-2]$. Indeed, by assuming that $T-e$ admits such an antimagic injection for a leave $e$ of $T$, there are $m-1$ values already taken by the labels of the edges in $T-e$, and $m-1$ edgesums which should be avoided for the edgesum of the vertex incident to $e$ in $T-e$ and for the leaf of $e$. Thus, if $3 m-2$ values are available, at least one of them must lead to an antimagic labeling of $T$. Such an inductive argument, however, cannot be applied to distance antimagic labelings.

Acknowledgements We are grateful to one of the referees for helpful comments and suggestions. 


\section{A final word}

Mirka Miller passed away in January 2016. This paper was written during successive meetings with Mirka in Jakarta, Bandung, Barcelona and Vientiane. After a long friendship full of mathematical discussions, this is the only mathematical paper that the two of us wrote together, not knowing that it would also be the last one. It is with a mixing of happiness and sorrow that I can eventually see it in print.

\section{References}

1. N. Alon, Combinatorial Nullstellensatz, Combinatorics, Probability and Computing 8, 729 (1999).

2. N. Alon, Additive Latin transversals, Israel J. Math. 117, 125-130 (2000).

3. N. Alon, G. Kaplan, A. Lev, Y. Roditty and R. Yuster, Dense graphs are antimagic, J. Graph Theory, 47(4), 297-309 (2004.

4. S. Arumugam, personal communication, 2012.

5. M. Bača and M. Miller, Super Edge-Antimagic Graphs: A Wealth of Problems and Some Solutions, BrownWalker Press, Boca Raton, Florida, USA, 2008.

6. B. Bollobás and O. Pikhurko, Integer sets with prescribed pairwise differences being distinct, European J. Combin. 26 (5), 607-616 (2005).

7. R. Bodendiek, G. Walther, On number theoretical methods in graph labelings, Res. Exp. Math. 21, 3-25 (1995).

8. P.D. Chawathe, V. Krishna, Antimagic labeling of complete $m$-ary trees, Number Theory and Discrete Mathematics, Trends Math., Birkhäuser, Basel, 77-80 (2002).

9. Y. Cheng, Lattice grids and prisms are antimagic, Theoretical Computer Science 374 (1-3), 66-73 (2007).

10. Y. Cheng, A new class of antimagic Cartesian product graphs, Discrete Math., 308(24), 6441-6448 (2008).

11. D. W. Cranston, Regular bipartite graphs are antimagic, J. Graph Theory, 60(3), 173-182 (2009) .

12. J. A. Gallian, A Dynamic Survey of Graph Labeling, The Electronic Journal of Combinatorics Dynamic Survey \# DS6 Eighteen Edition (2015).

13. N. Hartsfield, G. Ringel, Pearls in Graph Theory, Academic Press, INC., Boston, 108-109 (1994).

14. D. Hefetz, Anti-magic graphs via the Combinatorial NullStellenSatz, J. Graph Theory 50 (4), 263-272 (2005).

15. G. Kaplan, A. Lev and Y. Roditty, On zero-sum partitions and anti-magic trees, Discrete Math. 309, 2010-2014 (2009) .

16. R.N. Karasev and F.V. Petrov, Partitions of nonzero elements of a finite field into pairs, Israel J. Mathematics 192, 143-156 (2012).

17. A. Lladó, S.C. López, J. Moragas, Every tree is a large subtree of a tree that decomposes $K_{n}$ or $K_{n, n}$, Discrete Math. 310, 838-842 (2010).

18. M. Miller, C. Rodger, R. Simantujak, Distance magic labelings of graphs, Australasian Journal of Combinatorics 28, 305-315 (2003).

19. J. Ryan, O. Phanalasy, M. Miller and L. J. Rylands, On Antimagic Labeling for Generalized Web and Flower Graphs, In C. S. Iliopoulos and W. F. Smyth, editors. Proceeding of IWOCA10, London, UK, July 2010. Lecture Notes in Computer Science, 6460, 303-313 (2011).

20. T.-M. Wang, Toroidal grids are antimagic, Proc. 11th Annual International Computing and Combinatorics Conference, COCOON'2005, LNCS 3595, 671-679 (2005).

21. T.-M. Wang and C. C. Hsiao. On anti-magic labeling for graph products. Discrete Math. 308(16), 3624-3633 (2008)

22. Y. Zhang and X. Sun. The antimagicness of the Cartesian product of graphs. Theor. Comput. Sci. 410(8-10), 727-735 (2009). 